\title{
Calibration and monitoring of the ASTRI-Horn telescope by using the night-sky background measured by the photon-statistics ("variance") method
}

\section{A. Segreto*, O. Catalano, M. C. Maccarone, T. Mineo, A. La Barbera, for the CTA ASTRI Project ${ }^{\dagger}$}

INAF - Istituto di Astrofisica Spaziale e Fisica Cosmica di Palermo, via Ugo La Malfa 153,

Palermo, Italy

E-mail: segretodifc.inaf.it

\begin{abstract}
ASTRI-Horn is the Cherenkov telescope developed by INAF and operating in Italy on the slopes of Etna volcano. Characterized by a dual-mirror optical system and a focal plane covered by silicon photomultiplier sensors, ASTRI-Horn is a prototype of the telescopes proposed to form one of the pathfinder sub-arrays of the Cherenkov Telescope Array Observatory in Chile. The electronics of the ASTRI-Horn telescope, optimized to detect nanosecond burst of light, is not able to directly measure any continuous or slowly varying flux illuminating its camera. To measure the intensity of the night sky background (NSB) in the field of view of the telescope, the firmware of the ASTRI-Horn camera continuously performs the statistical analysis of its detector signals and periodically provides in output the "variance" of each pixel, which is linearly dependent on the rate of detected photons; in this way, an indirect, but accurate measurement of the NSB flux is obtained without interference with the normal telescope operation. In this contribution we provide an overview of several calibration and monitoring tasks that can be performed in a straightforward way by the analysis of the "variance" data such as the camera astrometry, the actual telescope orientation and the monitoring of its optical point spread function.
\end{abstract}

36th International Cosmic Ray Conference -ICRC2019-

July 24th - August 1st, 2019

Madison, WI, U.S.A.

\footnotetext{
${ }^{*}$ Speaker.

${ }^{\dagger}$ for consortium list see PoS(ICRC2019)1177
} 


\section{Introduction}

ASTRI-Horn [1] is the end-to-end prototype telescope developed by the Italian National Institute of Astrophysics, INAF, in the context of the Cherenkov Telescope Array Observatory, CTAO. It has been installed in Italy at the INAF station Fracastoro (located at Serra La Nave on Mount Etna) during Fall 2014. The ASTRI-Horn telescope is characterized by a dual-mirror optical system and a curved focal surface covered by silicon photomultiplier $(\mathrm{SiPM})$ sensors $\left(7 \times 7 \mathrm{~mm}^{2}\right)$ organized in photo detection modules (PDMs) managed by a fast front-end electronics based on the ASIC CITIROC [2].

In a way similar to other telescopes designed to detect fast pulsed signals (like e.g. the Fluorescence light or Cherenkov radiation produced by air showers), ASTRI-Horn adopts an AC-coupled readout electronics that provides several advantages like, e.g., the safe connection between electronic stages powered at different voltage levels and keeps the working points of the signal amplifiers and the baselines of the ADC converters stable at a predefined value, against any variation of the external background light.

The AC-coupling however, blocking any slow varying signal, makes the telescope blind to the Night Sky Background (NSB) flux (diffuse emission and stars) thus eliminating any direct feedback from the sky in the telescope field of view (FoV). However, due to the fact that the light is a sequence of photons described by a Poisson process, the individual electronic pulses generated at the random photon arrival times, produce fast varying fluctuations in the total electronic signal that are not blocked by the AC-coupling and, reaching the input of the ADC converter, add a noise component which is dependent on the rate of detected photons.

While the mean value of the ADC pedestal is therefore independent of the NSB flux, the variance (square of standard deviation) of the pedestal fluctuations, $\sigma^{2}$, is linearly dependent on it, being expressed by

$$
\sigma^{2}=\sigma_{\text {dark }}^{2}+\sigma_{\text {sky }}^{2}=\sigma_{\text {dark }}^{2}+k \cdot \Phi_{\text {sky }}
$$

where $\sigma_{\text {dark }}^{2}$ is the intrinsic variance of the electronics and the detector as it would be observed in perfectly dark conditions, and $\sigma_{\text {sky }}^{2}$ the variance induced by the external NSB flux which is directly proportional to the photon flux, $\Phi_{\text {sky }}$, illuminating the detectors.

This equation shows how measurements of the pedestal variance performed by the statistical analysis of a sequence of consecutive ADC converter outputs, allow us to obtain an indirect measure of night sky flux brightness without requiring any additional hardware. The statistical accuracy that can be obtained with this method depends on the number of ADC samples used for the analysis; denoting with $\delta \sigma^{2}$ the statistical uncertainty on the variance estimated from $N_{\mathrm{ADC}}$ samples, and assuming that the samples follow a normal distribution, its fractional uncertainty is expressed by the formula [3]

$$
\frac{\delta \sigma^{2}}{\sigma^{2}}=\sqrt{\frac{2}{N_{\mathrm{ADC}}-1}}
$$

From this equation we obtain that by using $N_{\mathrm{ADC}}=2^{16}$ samples, the variance of the pedestal noise is estimated with a statistical uncertainty of $\approx 0.55 \%$ and, assuming negligible the intrinsic dark noise, also the photon flux is estimated with the same accuracy level. 


\section{The ASTRI-Horn real time "variance" computation}

Using a method similar to the one implemented for the Fluorescence telescopes operating at the Pierre Auger Observatory [4, 5], an algorithm implemented in the ASTRI FPGA front-end board performs in real time, for each camera pixel, the sampling of the ADC baseline, and accumulates the sum of their values and of their squares; the algorithm runs in parallel with the scientific data taking discarding the samples acquired at the occurrence of camera triggers.

After a predefined number of successive ADC samples, the results of the accumulated data are inserted in the output telemetry and from these values, in a straightforward way, the "variance" maps of the focal plane are periodically obtained. Taking into account that the front end of the ASTRI-Horn electronics requires $16 \mu$ s to read-out the ADC samples of the whole detector plane, the total time required to produce a "variance" map with high statistical significance ( $2^{16}$ samples) is $\approx 1 \mathrm{sec}$, which is sufficient to monitor the temporal variations of the NSB flux.
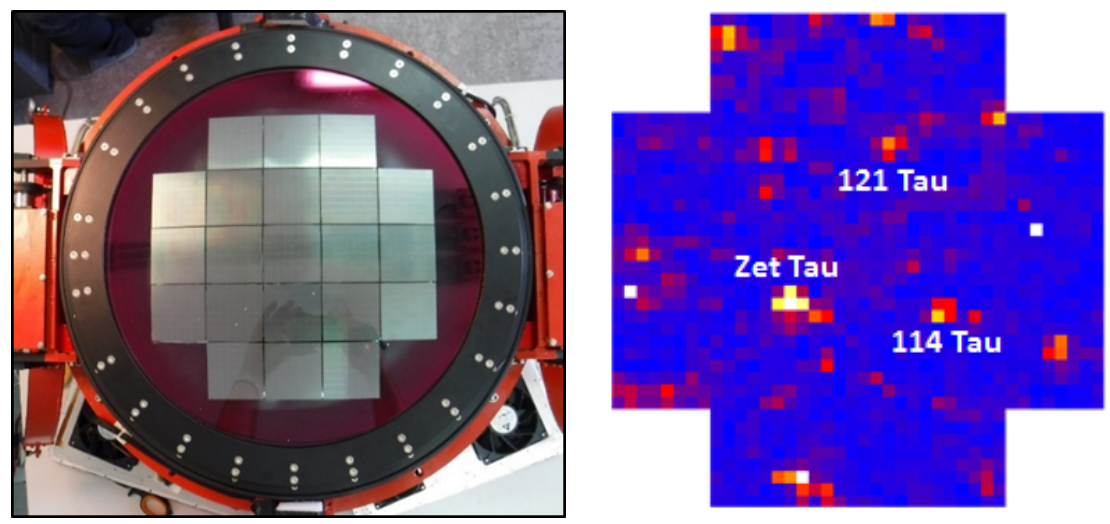

Figure 1: Left panel: the layout of the SiPM (64 pixels) tiles, each one integrated in a PDM (Photo Detection Module), covering the ASTRI curved focal plane. Right panel: an example of a single "variance" image obtained with the telescope pointing to the Crab, where the presence of several stars, although with a quite coarse pixel resolution, is evident.

In Fig. 1 we show an image of the ASTRI focal plane, where the detector layout is visible, and one example of "variance" acquisition, obtained while the telescope was pointing in the Crab direction, where it is well evident as several stars are clearly detected.

\section{Applications of the "variance" data}

There are numerous possible application of the NSB measured by means of the "variance" data: verification of the mirror segments alignment, astrometric calibration of the camera FoV, telescope pointing monitor, point spread function measurements, and much more[6]. In the following sections, using real data, we illustrate some of the problems that have been solved thanks to this innovative method.

\subsection{Verification of the alignment of primary mirror segments}

The first useful application obtained by the "variance" sky maps was the discovery of the misalignment of several segments of the ASTRI-Horn primary mirror. In Fig. 2, we show the 
sky map that was obtained with the telescope pointing to the "Orion's Belt" where the repetitive pattern of bright spots near the three brightest stars are a clear indication that several segments of the ASTRI-Horn primary mirror were not correctly aligned.

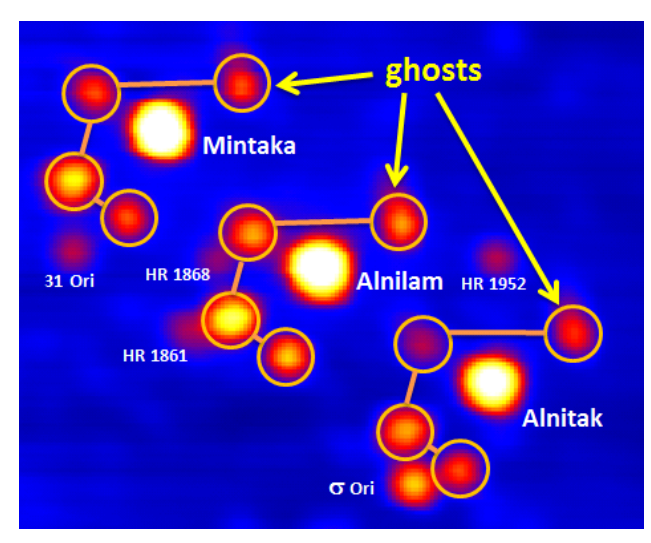

Figure 2: The first "variance" sky map obtained with the ASTRI-Horn telescope pointing to the "Orion's Belt" region; the numerous spots in repetitive pattern around the three brightest stars are caused by several tilted segments of the primary mirror.

Following this discovery, the realignment [7] of the mirror segments was performed by using, as feedback for the actuators, the optical images taken with a CCD camera temporarily put on the focal plane after removal of the ASTRI-Cherenkov camera. More conveniently, a similar procedure could be implemented by directly using the "variance" information from the Cherenkov camera as feedback for the actuators movements.

\subsection{Astrometric calibration of the camera FoV}

A second important application of the "variance" maps is the astrometric calibration of the camera FoV; this is done by keeping the telescope in staring mode (i.e. fixed azimuth. and elevation coordinates) and acquiring "variance" data for a time long enough that, thanks to the apparent rotation of the sky, numerous bright stars cross the telescope FoV in different positions.

The sky maps obtained by mosaicking the data acquired by multiple subsets of the camera pixels allow to determine their actual pointing coordinates and, combining all the information, to derive the actual camera plate scale, axes orientation and any eventual deformation of the FoV at large off-axis angles. For example, in Fig. 3 on the left panel, we show the sky maps (in equatorial R.A. - Dec. coordinates) obtained in $\approx 1$ hour of sky observation from some individual PDMs $(8 \times 8$ pixel $)$ with the telescope kept in a fixed position; the skymaps maps partially overlap each other since, as time passes, the same sky region is observed by multiple PDMs.

This procedure can be repeated with the telescope pointing in different azimuth and altitude angles, to verify if there is any modification of the FoV due to e.g. static deformation of the telescope structure caused by gravity; the results obtained can then be used to improve the telescope pointing model.

To show the sensitivity to star flux that can be reached with the "variance" method, in the right panel of Fig. 4 we show a sky map with the star identification and, on the left panel, an optical telescope image where the star visual magnitude is indicated; notwithstanding the quite low, 

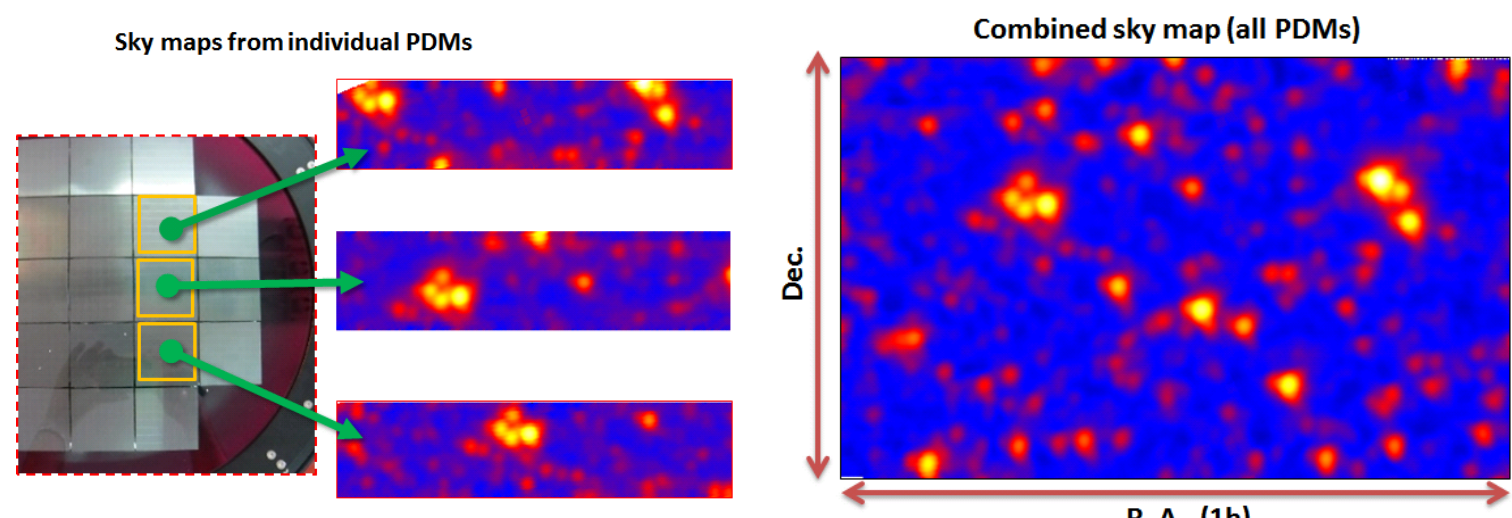

Figure 3: On the left, example of sky maps (in equatorial coordinates) generated by using, for each one, only data relative to individual ASTRI-Horn camera modules (PDM), acquired by keeping the telescope at fixed azimuth and elevation coordinates; several stars are visible in multiple sky maps since the horizontal axis is not only R.A. but also indicates the elapsed time of the measurement. On the right panel the sky map (Leo Minoris region) obtained by overlapping in R.A.-Dec. coordinates all the PDM images after astrometric calibration of the whole detector plane.

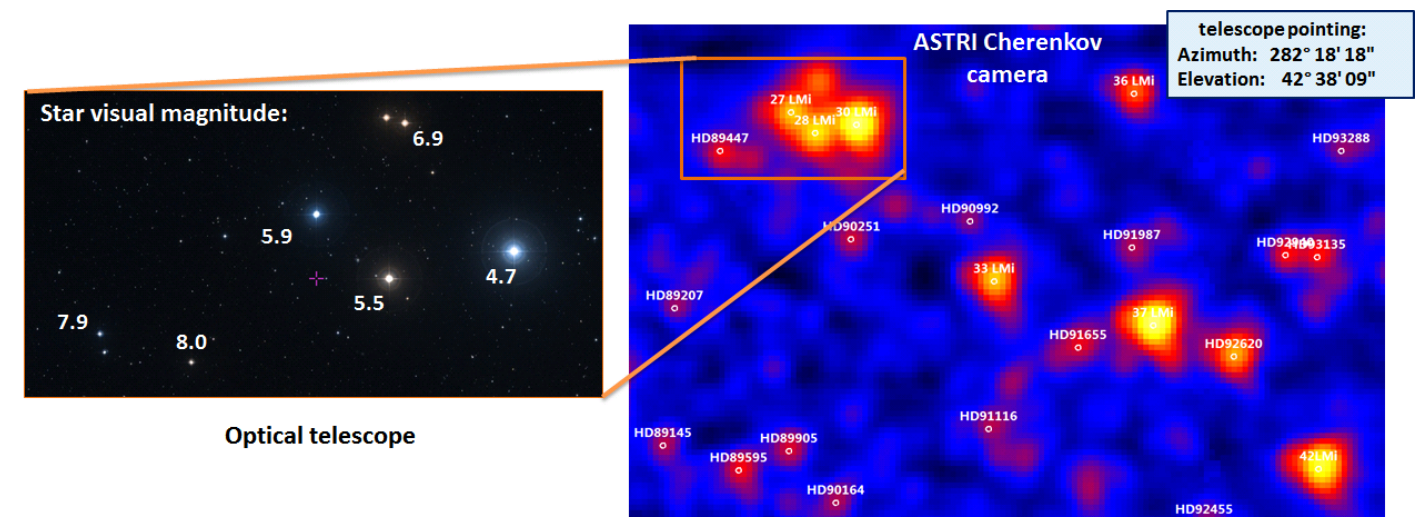

Figure 4: Right: sky map (equatorial R.A.-Dec. coordinates) where the expected stars positions are shown. On the left panel a sky map region as seen by an optical telescope were the stars visual magnitude is indicated.

$\approx 42^{\circ}$, elevation pointing angle in this observation (to which corresponds an elevated atmospheric attenuation) and the reduced mirror collecting area (as 2 mirror segments that could not be aligned were covered with black fabric), all known stars up to $8^{\text {th }}$ visual magnitude are clearly detected.

\subsection{Calibration and monitoring of the telescope pointing}

Another important application of the "variance" data is as auxiliary tool to monitor the telescope pointing during tracking observations. In fact, although a small CCD camera (PMC), placed on the support structure of the ASTRI-Horn secondary mirror, provides high angular resolution ( 7.25 arcsec per pixel) images of the sky where the telescope is pointing, its optical axis may not be precisely aligned with the geometric center of the Cherenkov camera.

To show an example of this problem observed on real data, Fig. 5 presents the sky map of the telescope FoV generated from the "variance" data acquired during a scientific observation tracking 


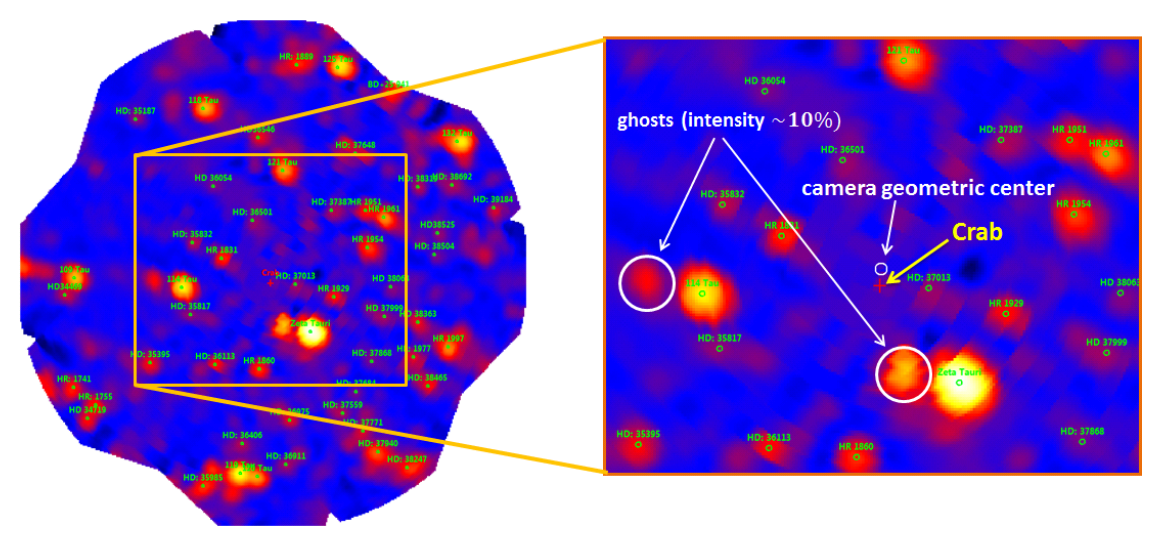

Figure 5: Left: the FoV sky map, in equatorial coordinates, obtained from the ASTRI-Horn "variance" data acquired simultaneously to a scientific observation tracking the Crab Nebula. Right: an enlarged view of the sky map center showing the offset between the actual Crab position and the point correspondent to the geometric center of the camera.

the Crab Nebula. While the Crab optical emission is too weak to be directly observed, the positions of the other stars in the FoV allow us to calibrate the sky map axes and therefore to locate the actual Crab position. As evident from the enlarged view shown in the right panel in Fig. 5, in this observation a significant offset $\left(\approx 10^{\prime}\right)$ was found between the Crab position and the sky coordinates correspondent to the geometric center of the camera (where it should have been located). In the next section present the method used to determine the origin of the observed pointing offset.

\subsection{Analysis of star trajectories in camera coordinates}
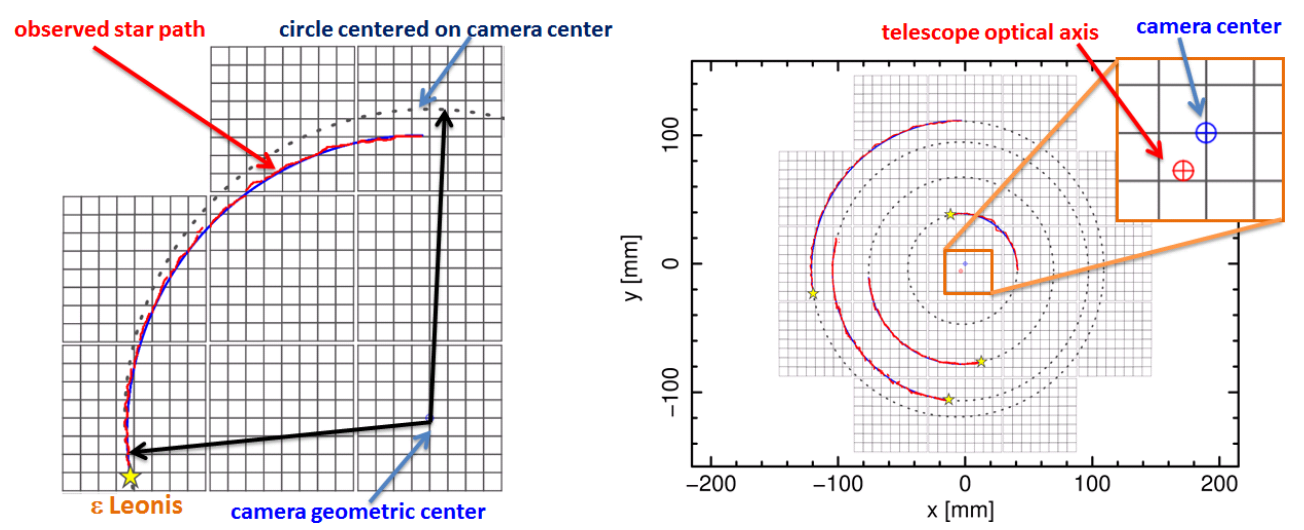

Figure 6: Left: the trajectory in camera coordinates of the star $\varepsilon$ Leonis as observed during a $\approx 4 \mathrm{~h}$ long tracking observation compared to the trajectory expected if the telescope optical axis were coincident with the camera center. Right: the path of 4 stars (which are concentric circles) observed during the observation, from which it is possible to determine the telescope axis offset.

The best way to individuate the origin of systematic pointing errors observed during tracking observations is to analyze the star trajectories in camera coordinates that, due to the field rotation effect, should be circles centered on the actual telescope pointing direction. For example, in Fig. 6, we show the star trajectories in $\mathrm{a} \approx 4 \mathrm{~h}$ long "Crab off-axis" observation; in the right panel we 
compare the trajectory of the brightest star in the camera FoV, $\varepsilon$ Leonis, to the circular trajectory that the star would have followed if the telescope pointing had been coincident with the geometric center of the camera.

The apparent increasing drift between the observed and the expected star positions is due to the fact that the telescope is tracking a sky coordinate that is not coincident with the camera center. Indeed, as shown in the left panel of Fig. 6, by the analysis of multiple star trajectories it is possible to localize the geometrical point corresponding to the telescope tracking direction with an accuracy that (depending on the number of stars and observing time) can reach a few arcsec level. The measured offset can therefore be inserted in the telescope pointing model to correct this systematic pointing error.

\subsection{Accuracy of using stars to monitor the telescope pointing}

Once the astrometric calibration of the camera FoV has been performed, we can use the star positions to periodically monitor the actual pointing direction of the telescope. To reduce the systematic errors in star localization due to the coarse pixel size of the Cherenkov camera (11.5 arcmin / pixel), it is necessary to simultaneously analyze the positions of several stars in the FoV and to average their position over a period of time long enough that they change appreciably their positions in camera coordinates, thanks to the field rotation.
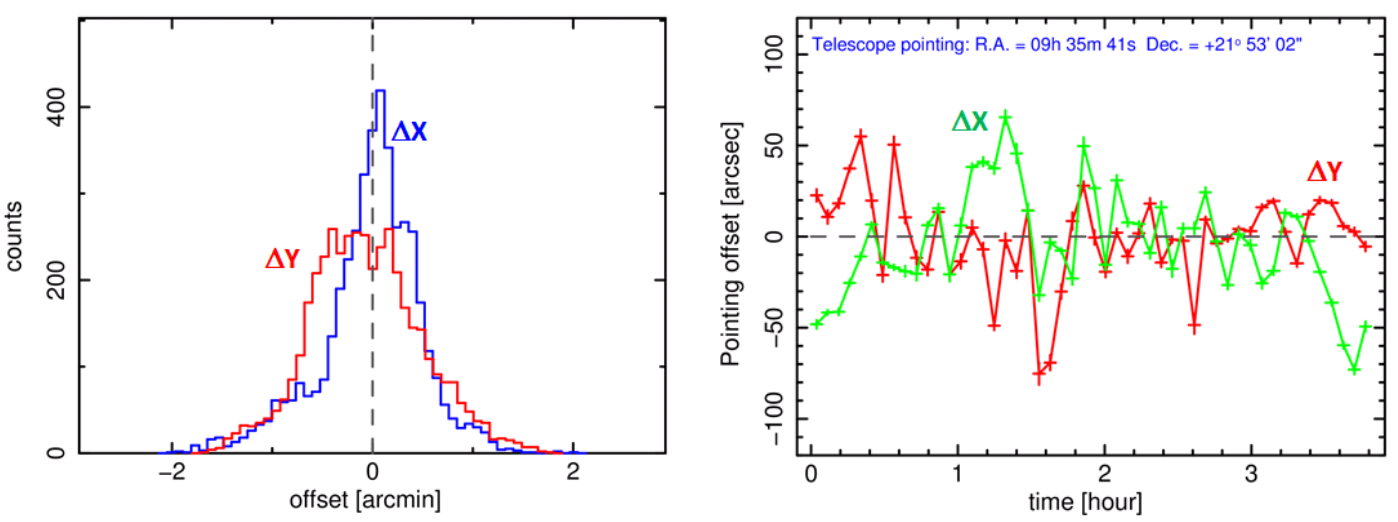

Figure 7: Left: statistical distribution of telescope pointing offsets measured in individual variance acquisition frames. Right: the temporal evolution of the telescope pointing offset as determined from the positions of a few stars in the FoV, averaged over 5 minutes.

As an example of the accuracy that can be obtained by the analysis of star positions, in the left panel of Fig. 7, we show the histograms of the offset between the nominal telescope pointing direction and the pointing estimated from the localization of the 4 brightest stars in individual "variance" sky maps acquired during a tracking observation. In the right panel of Fig. 7 we show the pointing offset obtained, as a function of time, by averaging the positions of the 4 stars over a 5 minutes observing interval; the results show how a few arcsec level accuracy can be reached in a few minutes.

\subsection{PSF monitoring}

During tracking observations all the stars move at a very slow speed (a few $\mu \mathrm{m} / \mathrm{sec}$ ) in camera coordinates, thus the "variance" of the pixels crossed by a star provide, with high spatial resolution, 
direct information on the optical point spread function (PSF); of course the curve observed as a function of the star position is not directly the profile of the optical PSF, but the total star light collected by the selected pixel. The actual shape of the optical PSF can be however easily recovered by means of analytical models or Monte Carlo simulation, whose parameters are tuned until a good match between the simulated and the observed star transit profiles are obtained.

As example, in the left panel of Fig. 8 we show the trajectory on the focal plane of a star observed during a tracking observation and, in the central panel, the temporal evolution of the "variance" of three pixels located along the star trajectory. The vertical dashed lines, indicating the expected transit times between adjacent pixels, are computed from the telescope pointing and camera geometry, and allow to verify the astrometric calibration of the FoV and the telescope pointing correction performed.
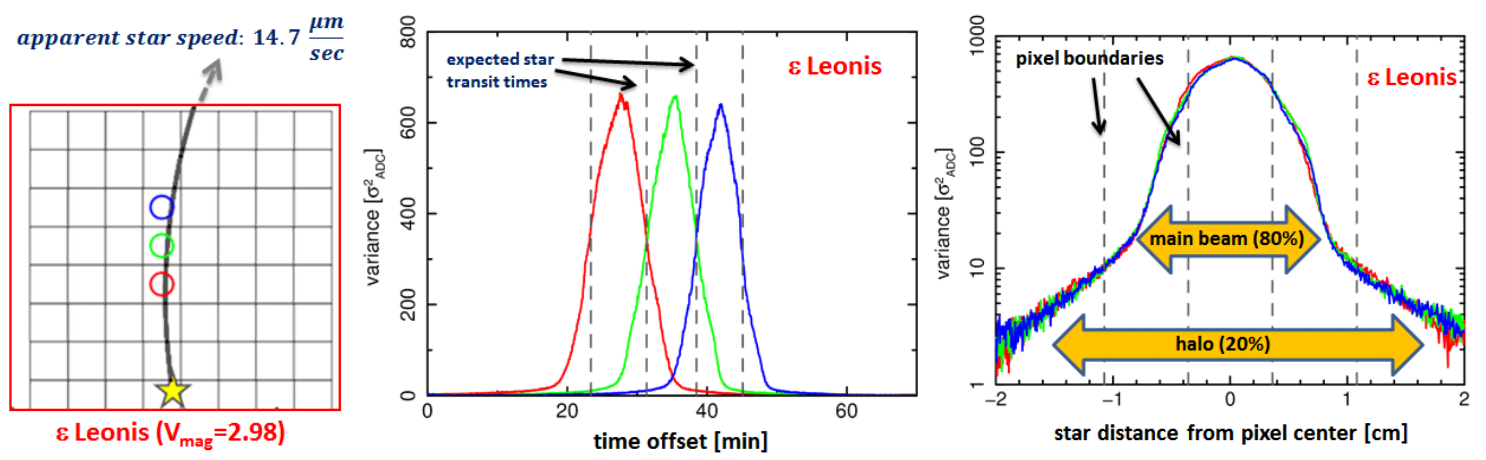

Figure 8: Left: trajectory on the focal plane of the star $\varepsilon$ Leonis during a tracking observation. Center: "variance" curves observed during the star transit over the three pixels. Right: transit profiles of the three pixels, as a function of the star offset from pixel centers, showing the significant degradation of the PSF observed after a volcanic eruption.

On the right panel, the curves of the three pixels are shown overlapped, in logarithmic scale, as a function of the offset of the star from the relative pixel center. It is evident in this acquisition, the presence on an extended halo (light scattered out of main beam) which is due to the highly degraded mirror conditions after a volcanic eruption. This example shows how, using the "variance data" it is possible to monitor in a straightforward way the PSF with a high dynamic range and a spatial accuracy by far superior to other methods.

\section{Conclusion}

We have presented the statistical method implemented in the FPGA logic of the ASTRI-Horn telescope that, by calculating the variance of the ADC pedestal noise in real time, is used to obtain optical maps of the sky in the telescope FoV with excellent accuracy and without requiring any additional hardware.

For the very first time, our measurements with the ASTRI-Horn telescope have demonstrated that, with a proper processing of the stars positions as directly observed from the Cherenkov camera it is possible, notwithstanding the coarse angular resolution of individual sky maps, to perform the full astrometric calibration of the telescope FoV and periodically monitor its pointing direction at a few arcsec level accuracy. 
Moreover, we have shown that, by using the signal generated by the stars during their transits over pixels, it is possible to monitor the evolution of the optical PSF with a spatial resolution and dynamical range by far superior to any other method.

\section{References}

[1] G. Pareschi, on behalf of the CTA ASTRI Project, The ASTRI program in the context of the Cherenkov Telescope Array Observatory, these proceedings

[2] O. Catalano, M.C. Maccarone, et al., The camera of the ASTRI SST-2M prototype for the Cherenkov Telescope Array, in proceedings of SPIE 9147, 91470D, 2014

[3] G. Casella, R. L. Berger, Statistical Inference, Duxbury Press, p. 331, 2002

[4] M. Kleifges, A. Menshikov, et al., Statistical Current Monitor for the Cosmic Ray Experiment Pierre Auger, IEEE Trans. Nucl. Sci, Vol. 50, No. 4, pp. 1204-1207, 2003

[5] A. Segreto, for the Pierre Auger Collaboration, Night Sky Background measurements by the Pierre Auger Fluorescence Detectors and comparison with simultaneous data from the UVscope instrument, in proceedings of 32nd ICRC-2011, 3, 129

[6] A. Segreto, for the Pierre Auger Collaboration, Using reference stars to verify the end-to-end absolute calibration and for the long term monitoring of the Fluorescence Detector telescopes at the Pierre Auger Observatory, in proceedings of CRIS 2018 (submitted)

[7] E. Giro, R. Canestrari, et al., First optical validation of a Schwarzschild Couder telescope: the ASTRI SST-2M Cherenkov telescope, A\&A 608, A86 (2017) 